\title{
Beam intensity effects in Fermilab Booster synchrotron
}

\author{
Jeff Eldred®, Valeri Lebedev@, Kiyomi Seiya, and Vladimir Shiltsev®* \\ Fermi National Accelerator Laboratory, P.O. Box 500, MS339, Batavia, Illinois 60510,USA
}

(Received 22 December 2020; accepted 19 March 2021; published 5 April 2021)

\begin{abstract}
Detrimental beam dynamics effects limit the performance of high-intensity rapid cycling synchrotrons (RCSs) such as the $8 \mathrm{GeV}$ proton Fermilab Booster. Here we report the results of comprehensive experimental studies of various beam intensity dependent effects in the Booster. In the first part, we report the dependencies of the Booster beam intensity losses on the total number of protons per pulse and on key operational parameters, such as the machine tunes and chromaticities. Then we cross check two methods of the beam emittance measurements (the MultiWires proportional chambers and the ionization profile monitors). Finally, we used the intensity dependent emittance growth effects to analyze the ultimate performance of the machine in the present configuration, with the maximum space-charge tuneshift parameter $\Delta Q_{\mathrm{SC}} \sim 0.6$, and after its injection energy is upgraded from 0.4 to $0.8 \mathrm{GeV}$.
\end{abstract}

DOI: 10.1103/PhysRevAccelBeams.24.044001

\section{INTRODUCTION}

High-energy, high-intensity proton beams are widely used for a broad spectrum of scientific research, including particle colliders, muon spectroscopy, crystallography of biological molecules, neutrino physics, and neutron scattering. Over the past four decades, the power of such beams has increased by about 3 orders of magnitude with an average doubling time of about four years [1,2]. Growing demands of the physics research call for more powerful, more productive and more sophisticated proton accelerators. For example, at present, the leading accelerators for neutrino research are the rapid cycling synchrotron (RCS) facilities J-PARC in Japan, which has reached $515 \mathrm{~kW}$ of the $30 \mathrm{GeV}$ proton beam power, and the Fermilab Main Injector, which delivers up to $800 \mathrm{~kW}$ of $120 \mathrm{GeV}$ protons, while the physics needs call for the next generation, higherpower, megawatt and multi-MW-class superbeam facilities [3].

Further progress in the proton beam power requires improvements in the accelerator technology [4] and addressing problems related to desired beam pulse structure, beam losses, and the lifetimes of beam targets [5-7]. Particularly challenging are issues associated with the beam dynamics, such as space-charge effects, instabilities, emittance growth, halo formation and losses, collimation, accumulation of secondary charges, linear and nonlinear

\footnotetext{
*shiltsev@fnal.gov
}

Published by the American Physical Society under the terms of the Creative Commons Attribution 4.0 International license. Further distribution of this work must maintain attribution to the author(s) and the published article's title, journal citation, and DOI. optics optimizations, etc.- - see comprehensive analyses in Refs. [8-12]. Advanced beam instrumentation, analytical methods and diagnostics [13-16], and experimental beam studies are critical for adequate understanding of the beam dynamics.

Here we present results of the experimental studies of high-intensity beam dynamics in the Fermilab $8 \mathrm{GeV}$ proton Booster RCS, which were carried out as part of the Summer 2019 Booster Studies program [17]. In Sec. II we give a brief description of the accelerator. Next, Sec. III is devoted to the beam loss diagnostics and measurements of the machine transmission efficiency and its dependence on the total number of protons per pulse, tunes and chromaticities. The beam emittance diagnostics and measurements, including analysis of the intensity dependent emittance growth, are presented in Sec. IV. Finally, we discuss general scaling laws and the ultimate performance of the machine now and after the upcoming upgrade in Sec. V.

\section{FNAL BOOSTER SYNCHROTRON}

The complex of Fermilab proton accelerators includes a $750 \mathrm{keV} \mathrm{H}^{-}$radiofrequency quadrupole (RFQ), $400 \mathrm{MeV}$ $H^{-}$pulsed normal-conducting if linac, $8 \mathrm{GeV}$ proton Booster synchrotron, $8 \mathrm{GeV}$ Recycler storage ring that shares a tunnel with the $120 \mathrm{GeV}$ proton Main Injector synchrotron, and a $3.1 \mathrm{GeV}$ muon delivery ring $[18,19]$. About $16 \mathrm{~km}$ of beam lines connect the accelerators and bring the beams to fixed targets and experiments for highenergy particle physics research at the Intensity Frontier. There are plans to further increase the facility power from the current world-leading level of $\sim 800 \mathrm{~kW}$ of average $120 \mathrm{GeV}$ beam power on the neutrino target to over $1.2 \mathrm{MW}$ at the start of the LBNF/DUNE experiment 
[20] in the second half of the 2020s via replacement of the existing $400 \mathrm{MeV}$ normal-conducting linac with a modern cw-capable $800 \mathrm{MeV}$ superconducting rf linear accelerator (PIP-II, see [21]) and corresponding beam line for injection into the Booster. There are also several concepts to further double the beam power to $>2.4 \mathrm{MW}$ after replacement of the existing $8 \mathrm{GeV}$ Booster synchrotron [22,23].

The Fermilab Booster [24] is a $474.2 \mathrm{~m}$ circumference, alternating-gradient, rapid-cycling synchrotron containing 96 combined-function magnets; see Fig. 1. Together with capacitor banks, these magnets form a resonant network $(Q=40)$ and get excited with a $15-\mathrm{Hz}$ biased sine wave. Beam acceleration ramp from $0.4 \mathrm{GeV}$ at injection to $8.0 \mathrm{GeV}$ at extraction is $33.3 \mathrm{~ms}$ long - half of the magnet cycle period-and contains about 20,000 turns. Correspondingly, all the parameters of the machine and beam significantly vary in the cycle from the currents in all correctors (trim dipoles, trim quads and skew quads, sextupoles and octupoles) to rf frequency, voltage and phase (see Fig. 2), from the betatron and synchrotron tunes and chromaticities to proton beam intensity, positions, sizes, emittances, bunch length and energy spread, etc. The main parameters of the Booster are given in Table I. Without going into details of the Booster high-intensity operation and interface with other machines in the complex, which can be found in Refs. [25-27], here we only briefly outline the main processes which occur at injection, transition crossing and extraction.

The Booster receives $400 \mathrm{MeV} \mathrm{H}$ beam of $201 \mathrm{MHz}$ bunches from the linac while it is close to the minimum of the magnetic field ramp. $H^{-}$particles are stripped of two electrons when they pass through a thin foil, and resulting protons are accumulated over many turns in the ring (the scheme known as charge exchange injection [28,29]).

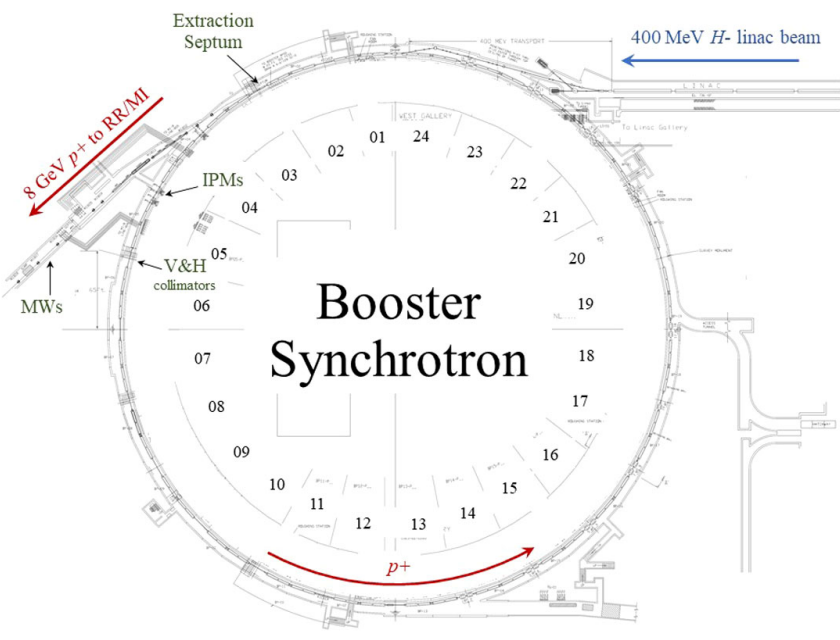

FIG. 1. Schematics of the Fermilab Booster synchrotron. Sectors, each consisting of four combined function magnets, are numerated 1 to 24 . Indicated are locations of the ionization profile monitors (IPMs), MultiWires (MWs) beam profile monitors in the $8 \mathrm{GeV}$ proton transport line and the $400 \mathrm{MeV} \mathrm{H}^{-}$ beam injection line.

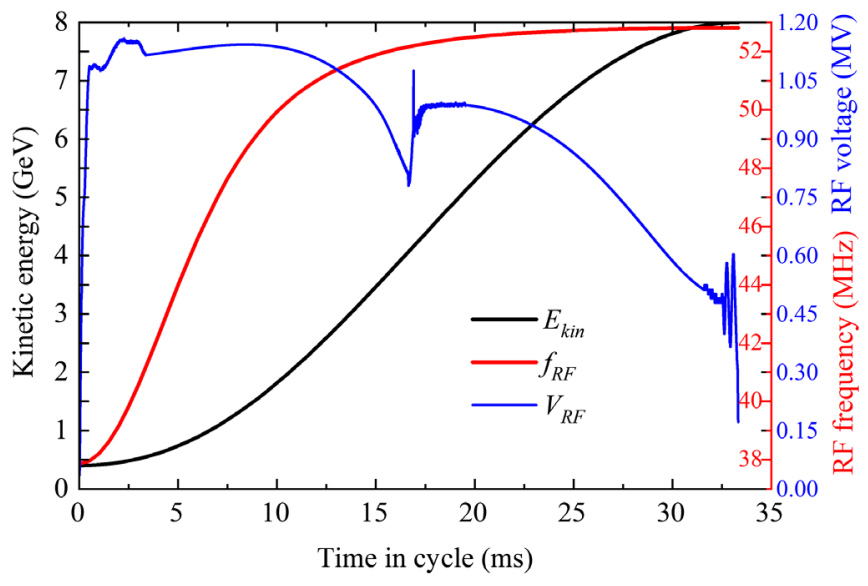

FIG. 2. Booster ramp: kinetic energy (black), rf voltage (blue) and frequency (red).

Correspondingly, the total injected and accelerated beam intensity $N_{p}$ scales with the linac current, which is typically $\sim 25 \mathrm{~mA}$, and the total number of injection turns $N_{\text {turns }}$, approximately as $N_{p}=0.34 \times 10^{12} \cdot N_{\text {turns }}$, e.g., about $4.8 \times 10^{12}$ for a typical 14-turn injection. The duration of the beam injection also scales with the number of turns as $2.2 \cdot N_{\text {turns }}[\mu \mathrm{s}]$. The beam is injected with rf voltage close to zero and is then adiabatically captured over about $300 \mu$ s by the rf system [30,31]. Right after injection and in the following several milliseconds, the high-intensity proton beam is subject to the strongest space-charge forces, characterized by the space-charge parameter $\Delta Q_{\mathrm{SC}} \geq 0.5$ (see below). Transverse and longitudinal ring impedances

TABLE I. Main operational parameters of the Fermilab Booster. $\beta_{p}$ and $\gamma_{p}$ are relativistic Lorentz factors of protons.

\begin{tabular}{|c|c|c|}
\hline Parameter & & Comments \\
\hline Circumference, $C$ & $474.20 \mathrm{~m}$ & \\
\hline $\begin{array}{l}\text { Injection energy } \\
\text { (kinetic), } E_{i}\end{array}$ & $400 \mathrm{MeV}$ & $\begin{array}{l}\beta_{p}=0.701 \\
\gamma_{p}=1.426\end{array}$ \\
\hline $\begin{array}{l}\text { Extraction energy } \\
\quad \text { (kinetic), } E_{f}\end{array}$ & $8 \mathrm{GeV}$ & $\begin{array}{l}\beta_{p}=0.994 \\
\gamma_{p}=9.526\end{array}$ \\
\hline $\begin{array}{l}\text { Cycle time, } \\
\qquad T_{0}=1 / f_{0}\end{array}$ & $1 / 15 \mathrm{~s}$ & 20,000 turns \\
\hline Harmonic number & 84 & \\
\hline $\mathrm{rf}$ frequency, $f_{r f}$ & $\begin{array}{l}\text { 37.77- } \\
52.81 \mathrm{MHz}\end{array}$ & injection-extraction \\
\hline $\begin{array}{l}\text { Maximum rf } \\
\text { voltage, } V_{r f}\end{array}$ & $1.1 \mathrm{MV}$ & \\
\hline $\begin{array}{l}\text { Transition } \\
\quad \text { energy, } E_{t r}\end{array}$ & $4.2 \mathrm{GeV}$ & $\begin{array}{c}\gamma_{t r}=5.478, \text { at } \\
t=17 \mathrm{~ms}\end{array}$ \\
\hline No. of cells, magnets & 24,96 & $\begin{array}{c}\text { FOFDOOD }, 96^{\circ} \\
\text { cell }\end{array}$ \\
\hline Total intensity, $N_{p}$ & $4.5 \times 10^{12}$ & $N_{b}=81$ bunches \\
\hline $\begin{array}{l}\text { rms normalized emittance, } \\
\varepsilon_{x, y}\end{array}$ & $2.0 \pi \mu \mathrm{m}$ & $12 \pi \mu \mathrm{m}$ for $95 \%$ \\
\hline$\beta$ functions, $\beta_{x, y}$ & $33.7 / 20.5 \mathrm{~m}$ & maximum \\
\hline Dispersion function, $D_{x}$ & $3.2 \mathrm{~m}$ & maximum \\
\hline
\end{tabular}



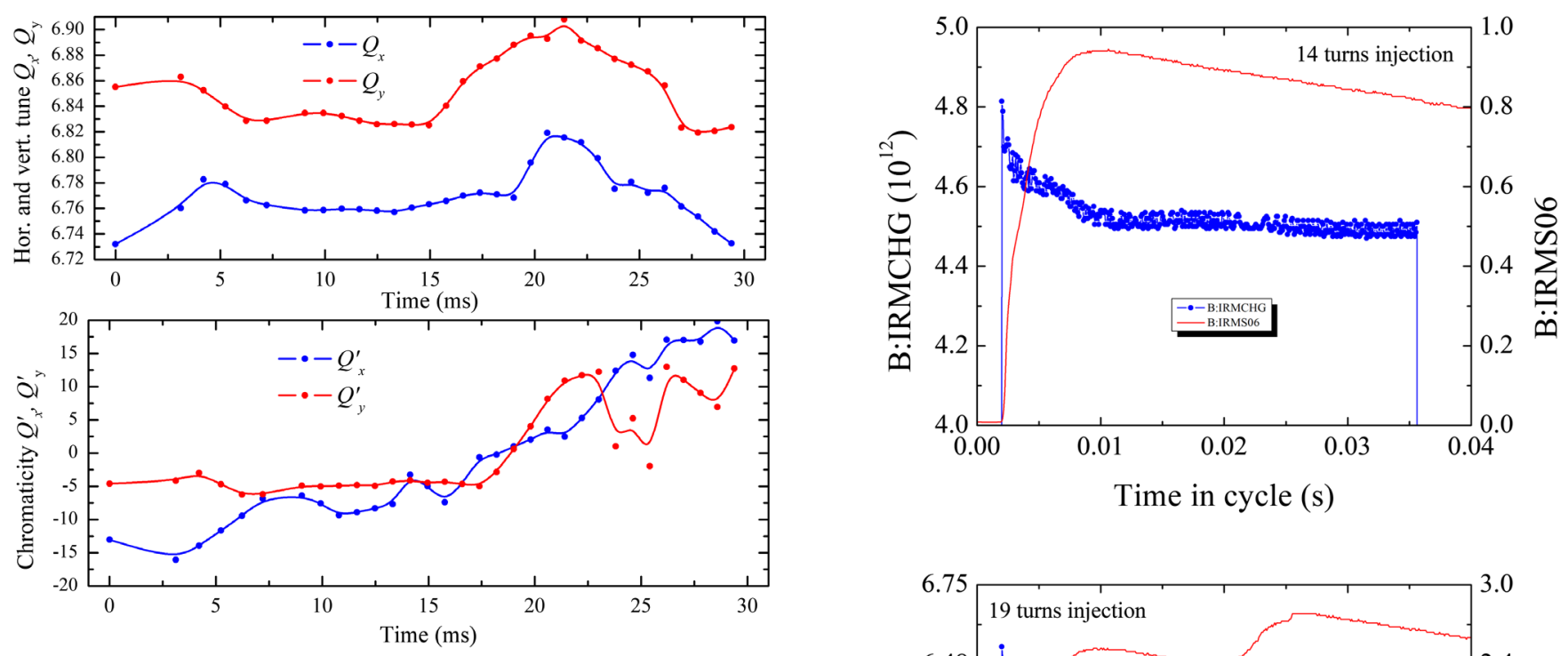

FIG. 3. Measured Booster beam characteristics during the acceleration cycle: (top) the horizontal (blue) and vertical (red) betatron tunes $Q_{x, y}$; (bottom) the horizontal (blue) and vertical (red) chromaticities $Q_{x, y}^{\prime}$.

are large $[10,32,33]$ and the Booster operation requires simultaneous, fast and idiosyncratic adjustment of orbits, optical functions [34], tunes, chromaticities and many other machine parameters on top of changing energy, rf voltage and frequency (see Fig. 3).

The transition occurs at about $17 \mathrm{~ms}$ into the Booster cycle (after injection), at the beam energy of $4.2 \mathrm{GeV}$. The Booster is currently operated without a dedicated $\gamma_{t}$-jump system, though the current of trim quadrupoles, rf system voltage and $\mathrm{rf}$ frequency curves have been tuned to minimize the losses and control the longitudinal emittance, which grows somewhat from its initial $95 \%$ value of about $0.08 \mathrm{eVs}$ [35-37].

The rapid acceleration in the Booster requires large accelerating voltage. To inject the Booster beam efficiently into the Recycler for slip stacking, it is desirable to rotate the beam in longitudinal phase space so as to reduce the momentum spread [38]. The Booster bunch rotation is performed via quadrupole excitation of the synchrotron oscillation as the rf voltage is modulated at twice the synchrotron frequency, and this drives a longitudinal quadrupole resonance. Once the beam energy is close to the extraction energy, we perform snap bunch rotation - i.e., at about $2 \mathrm{~ms}$ before the end of the cycle, the rf voltage is increased slowly to $650 \mathrm{kV}$ to increase the energy spread of the bunches and then dropped down rapidly to $130 \mathrm{kV}$. This gives the required small energy spread for the beam for slip stacking in the downstream accelerators.

The overall average Booster beam loss limit has been administratively set to $W=525 \mathrm{~W}$, i.e., $35 \mathrm{~J}$ per cycle with $15 \mathrm{~Hz}$ beam cycles. Such a level allows us to maintain all elements in the Booster tunnel without excessive radiation

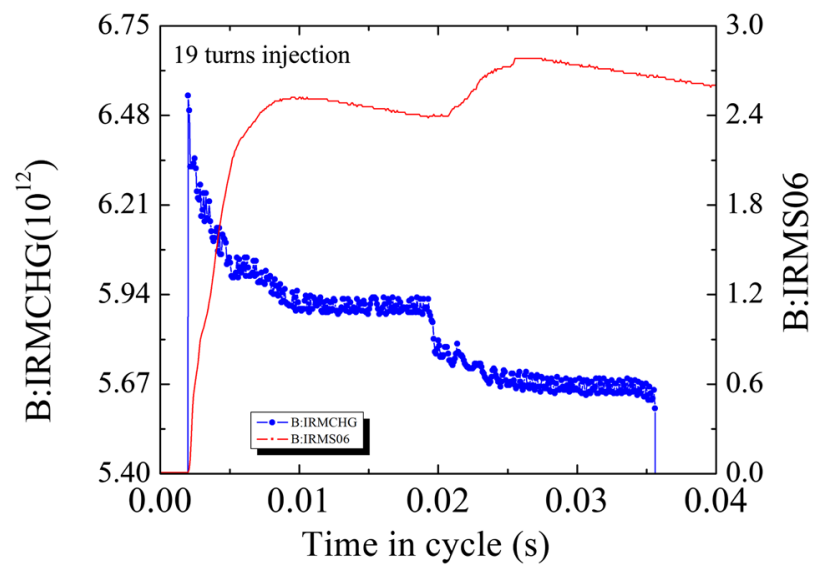

FIG. 4. Booster beam losses in the acceleration cycle: (top) the BCHG0 intensity monitor signal (blue, left axis) and the S06 beam loss monitor (BLM) readings (red, right axis) for nominal operational 14 turns injection intensity; (bottom) the same for higher intensity 19 turns injection.

exposure and corresponds to either $13 \%$ beam loss at injection energy or $1.2 \%$ at the transition, or $0.6 \%$ at extraction for nominal intensity of about $4.5 \times 10^{12}$ protons per pulse. As illustrated in Fig. 4, at nominal intensity, with 14 turns injection, the losses mostly occur at injection, but the loss at transition becomes dominant at higher intensities. In general, the beam loss induced radiation is the most important and most challenging factor limiting the performance of high-intensity RCSs [4].

\section{BEAM LOSSES}

\section{A. Beam loss diagnostics}

The main diagnostic of the total circulating Booster beam intensity is the ACNET [39] (Fermilab global accelerator control system) channel B:CHG0, which uses the signal from the beam current toroid properly mixed and averaged with the rf waveform signal. The raw B:CHG0 toroid intensity data are quite reliable and accurate to better than a fraction of one percent at relatively stable machine 
and beam parameters, but require correction of the systematic effects early in the cycle, when the rf frequency, the bunch length and the bunch structure are quickly changing in time. The required correction was established using a cross calibration of the intensity loss $\Delta N_{p}$ reported by B:CHG0 and the power loss signal measured by the most appropriate beam loss monitor BLMS06-see Fig. 4. The BLM at S06 is located between two Booster collimators, and in regular operation it registers the most significant loss flux over the cycle. The BLM signal represents an integral of a proportional chamber with decay time of $\sim 200 \mathrm{~ms}$. This signal is reset at the beginning of each cycle. As one can see, BLMS06 peaks for the first time at $10 \mathrm{~ms}$ into the cycle with the amplitude proportional to the power loss integrated well over the initial lossy period of the Booster cycle. The loss monitor signals at $10 \mathrm{~ms}$ were compared to the reported changes in the toroid signal B: $\mathrm{CHGO}$, and it was found that the latter overestimates $O(5 \%)$ losses at small intensities by about $2 \%$, but that difference disappears (mutual linearity gets restored) at higher intensities and higher fractional losses-see details in [40].

\section{B. Fractional beam loss}

Our studies reveal three kinds of Booster beam intensity losses: (1) minor intensity-independent loss at injection $\sim(1-2) \%$ due to the so-called "notch clearing"; (2) significant $O(5 \%)$ loss shortly after the injection that can be attributed to space-charge effects as it strongly depends on the intensity, the working point tunes and chromaticities; and (3) thresholdlike increase of the losses $O(5 \%)$ during and after the transition above $N_{p}=6 \times 10^{12}$.

The notch clearing. - The Booster extraction kicker rise time is about $70 \mathrm{~ns}$ long, and to reduce the losses at extraction one requires an empty gap in the beam structure. The beam gap is created by removing three out of 84 possible bunches at the lowest feasible beam energies. First, a recently built laser system [41] is used to create the notch within a linac beam pulse immediately after the RFQ at $750 \mathrm{keV}$, where activation issues are absent. The beam with such a gap is injected into the Booster. The laser notcher system is not $100 \%$ efficient and, in addition, some particles slip into the gap before the injection rf capture, and as a result there is $400 \mathrm{MeV}$ beam in the gap as illustrated by Fig. 5. These particles are cleared out at approximately 150 turns after the injection by a kicker pulse that removes $1.4 \pm 0.4 \%$ of the total beam intensity. The particles are directed to a special in-line beam dump which intercepts them with an efficiency much better than the efficiency of the collimation system, so these losses are excluded from the following analysis of the intensity dependent effects. Ongoing improvements to the laser power and optical system are expected to greatly reduce the number of unwanted particles in the notch gap.

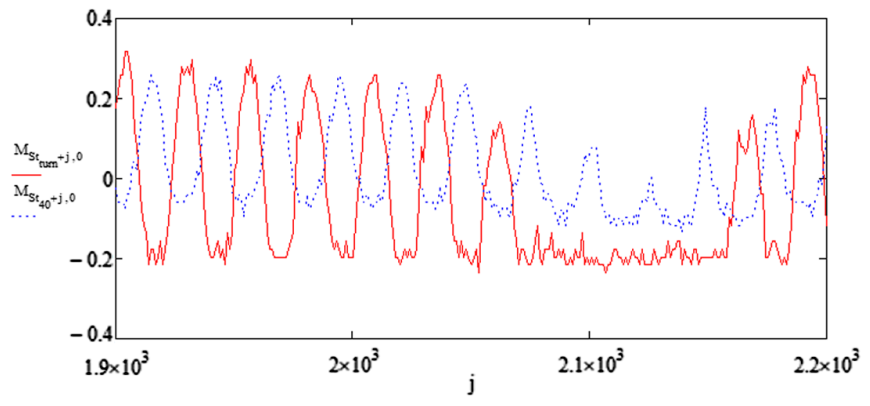

FIG. 5. Booster resistive wall monitor traces sampled every $1 \mathrm{~ns}$ (index $j$ ) for the bunch beam current profiles right before (dashed blue) and 40 turns after (solid red) the extraction gap clearing.

The largest, and of the most operational concern, intensity dependent losses which take place over the first $8 \mathrm{~ms}$ after the injection are presented in Fig. 6. As one can see, the losses quickly grow with $N_{p}$-solid line in Fig. 6 is for the fit

$$
\frac{\Delta N_{p}}{N_{p}}=0.01+0.07 \cdot\left(\frac{N_{p}}{7 \times 10^{12}}\right)^{3} .
$$

The nonzero intercept at small $N_{p}$ might indicate either a different mechanism of constant losses at low intensity or an insufficient measurement accuracy.

The beam intensity losses at the transition crossing were found to be below $\lesssim 0.5 \%$ up to a threshold at about $N_{p}=$ $6 \times 10^{12}$ and to then quickly reach $7 \%$ at $N_{p}=7 \times 10^{12}$ [40]. The characteristic dependence and underlying physics mechanism is quite different from what is observed at the injection. The intensity dependence at transition is most likely related to the longitudinal beam loading and the voltage induced by large longitudinal impedance of the Booster laminated vacuum chamber interior [32,36,42]. We also have to note that, for optimal transmission efficiency, the transition crossing has to be retuned for higher

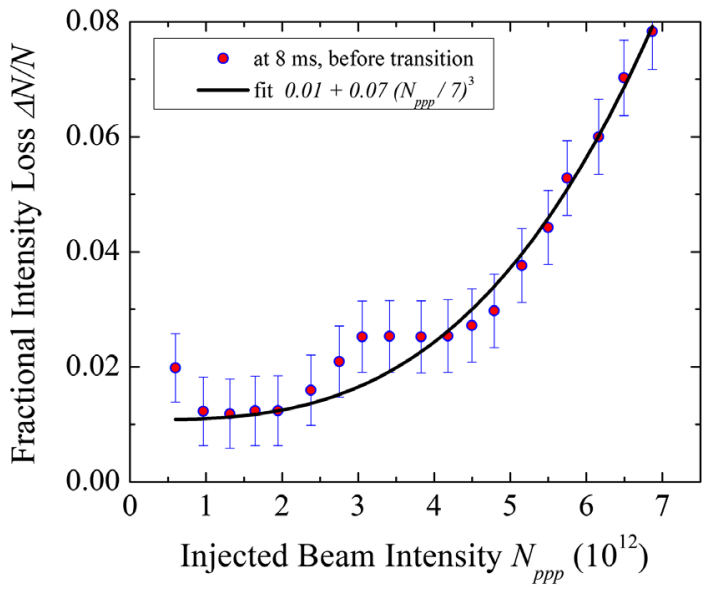

FIG. 6. Intensity-dependent fractional Booster beam intensity loss at injection vs total number of protons. 
intensities, while during our studies we operated with the rf and other machine parameters at the transition optimized for the nominal operational intensity of $N_{p}=4.5 \times 10^{12}$.

Altogether, in our study the fractional beam intensity losses over the entire Booster cycle are about $4.2 \pm 0.5 \%$ for the nominal intensity, while at the record high injected intensity $N_{p}=7 \times 10^{12}$ they are about $15 \%$. Obviously, such losses are not acceptable for routine operation within the administrative beam power loss limit.

\section{Tune scans}

To better understand the nature of the intensity loss phenomena, we have studied the Booster transmission efficiency dependence on the chromaticities and tunesthe $Q_{x, y}^{\prime}$ and $Q_{x, y}$ scans. Figure 7 shows the dependence of the losses over the first $1 \mathrm{~ms}$ of the Booster cycle $(\sim 450$ turns) at the nominal operational tunes $Q_{x, y}=6.78 / 6.88$, but at three different chromaticity settings - the nominal one $Q_{x, y}=-16 /-4$, and then at $Q_{x, y}=-12 /-12$, and at $Q_{x, y}=-20 /-20$. The fractional losses were calculated out of the B:CHG0 signal, corrected for the systematic error at lower intensities and with the extraction gap clearing loss subtracted, following the method presented in the preceding section.

The results presented in Fig. 7 clearly show significant increase of the losses with the chromaticity. Taking for simplicity the same functional dependence on intensity as in Eq. (1), the chromatic dependence of the loss data is consistent with

$\frac{\Delta N_{p}}{N_{p}}=0.013+0.10 \cdot\left(\frac{N_{p}}{7 \times 10^{12}}\right)^{3}\left(\frac{Q_{\mathrm{avg}}^{\prime}}{10}\right)^{1.9 \pm 0.2}$,

with $Q_{\mathrm{avg}}=\left(\left|Q_{x}^{\prime}\right|+\left|Q_{y}^{\prime}\right|\right) / 2$ denoting the average chromaticity. There is a lower limit on operational chromaticity

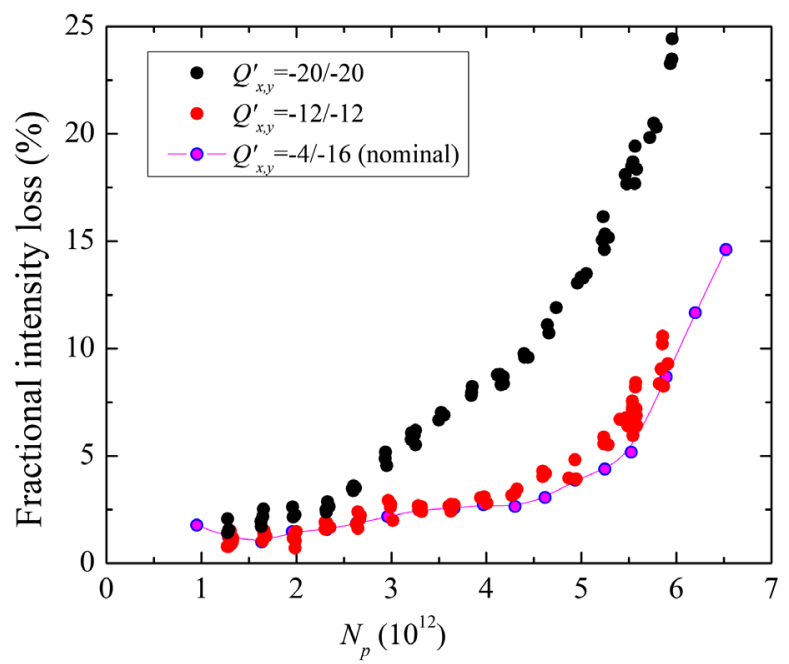

FIG. 7. Transmission efficiency $1 \mathrm{~ms}$ after injection for different chromaticities $Q_{x, y}^{\prime}$ vs $N_{p}$. that depends on the intensity and is usually associated with the need to maintain the coherent beam stability. Correspondingly, the low chromaticity operation is possible only at low intensities. Notably, the strong dependence of the losses at injection on the chromaticity, presumably due to the space-charge effects, $\Delta N_{p} \propto Q^{\prime 2}$, is similar to the incoherent beam losses due to parasitic beam-beam effects observed in the Tevatron collider [43].

The tune scans were carried out under six different conditions: (i) low intensity and low chromaticity: $N_{p}=0.95 \times 10^{12}, Q_{x, y}^{\prime}=-6 /-6$; (ii) low intensity and high chromaticity: $N_{p}=0.95 \times 10^{12}, Q_{x, y}^{\prime}=-20 /-20$; (iii) high intensity and medium chromaticity: $N_{p}=4.3 \times 10^{12}, Q_{x, y}^{\prime}=-16 /-4$; (iv) high intensity and high chromaticity: $N_{p}=4.3 \times 10^{12}, Q_{x, y}^{\prime}=-20 /-20$; (v) medium intensity and medium chromaticity: $N_{p}=2.6 \times 10^{12}, Q_{x, y}^{\prime}=-12 /-12$; (vi) medium intensity and high chromaticity: $N_{p}=2.6 \times 10^{12}, Q_{x, y}^{\prime}=-20 /-20$. Note that vertical and horizontal tunes and chromaticities varied only for the time period of $2 \mathrm{~ms}$ after the injection, and for the rest of the Booster cycle they stayed the same as they were for the routine operational cycles.

The results of the first four are presented in Fig. 8. One can see that, in general, an increase of either the chromaticity or intensity or both leads to reduction of the available tune space for low loss operation and generally lower optimal transmission efficiencies. Table II summarizes the findings.

The tune scan data reveal stronger sensitivity of the losses to the vertical tune than to the horizontal one. For example, a 14-units increase of the chromaticity from -6 to
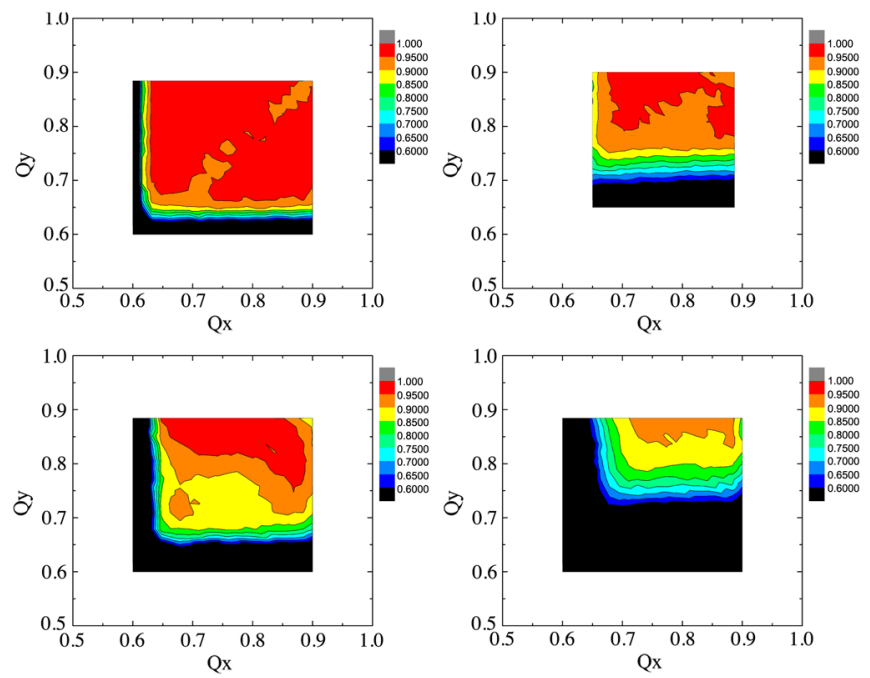

FIG. 8. Tune scans of the transmission efficiency over the first millisecond after injection: (a) (top left) at the $N_{p}=0.95 \times 10^{12}$ and $Q_{x, y}^{\prime}=-6 /-6$; (b) (top right) $N_{p}=0.95 \times 10^{12}$ and $Q_{x, y}^{\prime}=-20 /-20$; (c) (bottom left) $N_{p}=4.3 \times 10^{12}$ and $Q_{x, y}^{\prime}=-16 /-4$; (d) (bottom right) $N_{p}=4.3 \times 10^{12}$ and $Q_{x, y}^{\prime}=-20 /-20$. 
TABLE II. Optimal working points for various injected intensities and injection chromaticities: the first line in each box is the minimal intensity loss $1 \mathrm{~ms}$ after injection (raw B:CHG0 data, uncorrected for the B:CHG0 systematic errors and the notcher gap cleaning) and the optimal horizontal and vertical tunes $Q_{x} / Q_{y}$; the second line is the same for the entire Booster cycle (at extraction).

\begin{tabular}{lcll}
\hline \hline$N_{p}, 10^{12}$ & $Q_{x, y}^{\prime}=-6 /-6$ & $Q_{x, y}^{\prime}=-12 /-12$ & $Q_{x, y}^{\prime}=-20 /-20$ \\
\hline 0.95 & $1.5 \%, 0.68 / 0.84$ & & $1.2 \%, 0.69 / 0.88$ \\
& $3.5 \%, 0.82 / 0.81$ & & $2.6 \%, 0.77 / 0.88$ \\
4.3 & & $3.0 \%, 0.74 / 0.87$ & $7.0 \%, 0.77 / 0.88$ \\
& & $7.0 \%, 0.80 / 0.90$ & $16 \%, 0.77 / 0.88$ \\
\hline \hline
\end{tabular}

-20 at $N_{p}=0.95 \times 10^{12}$ resulted in the reduction of the $90 \%$ transmission tune area by $d Q_{y}=0.05$ in the vertical plane and only $d Q_{x}=0.02$ in the horizontal plane; see Figs. 8(a) and 8(b). Similarly, the change of the chromaticity from -12 to -20 for $N_{p}=4.3 \times 10^{12}$ led to shrinkage of the $90 \%$ transmission tune area by $d Q_{y}=$ 0.1 and $d Q_{x}=0.05$, as depicted in Figs. 8(c) and 8(d). That is indicative of a stronger resonance in the vertical than the horizontal.

\section{BEAM EMITTANCE EVOLUTION}

\section{A. Beam emittance diagnostics}

In the Booster, there are two types of instruments to measure beam sizes and, therefore, transverse emittances: the MultiWires (MWs) and the ionization profile monitors (IPMs).

Vertical and horizontal MWs are installed in the extraction beam line and, therefore, can measure only the emittances of the extracted Booster beam. There are 48 wires in each instrument, spaced by $1 \mathrm{~mm}$. The focusing optics functions at the MWs location are $\beta_{x}=16.2 \mathrm{~m}$, $\beta_{y}=25.9 \mathrm{~m}$ and $D_{x}=-1.65 \mathrm{~m}$. Statistical $\mathrm{rms}$ error of the MWs emittance measurement is about $0.05 \mathrm{~mm}$ mrad.

IPMs operate by collecting ions created after the ionization of residual vacuum molecules by high-energy charged particle beams $[14,16]$, which are then guided to a detector by a uniform external electric field $E_{\text {ext }}$. The detector consists of many thin parallel strips, whose individual signals are registered to make the beam profile signal ready for processing. Two IPMs vertical and horizontal are installed in the Booster at the location with $\beta_{x}=6.0 \mathrm{~m}$, $\beta_{y}=20.3 \mathrm{~m}$ and $D_{x}=1.8 \mathrm{~m}$ [44]. The electric field of about $2.4 \mathrm{kV} / \mathrm{cm}$ is formed by application of $V=24 \mathrm{kV}$ extracting voltage over a $D=103 \mathrm{~mm}$ gap. The MCPbased ion detector employs an array of parallel thin anode strips spaced $1.5 \mathrm{~mm}$ apart. IPMs are very fast and report the average rms beam sizes (determined by the Gaussian fits of the profiles) on every Booster turn. Note that the Booster IPMs do not employ external magnetic fields to

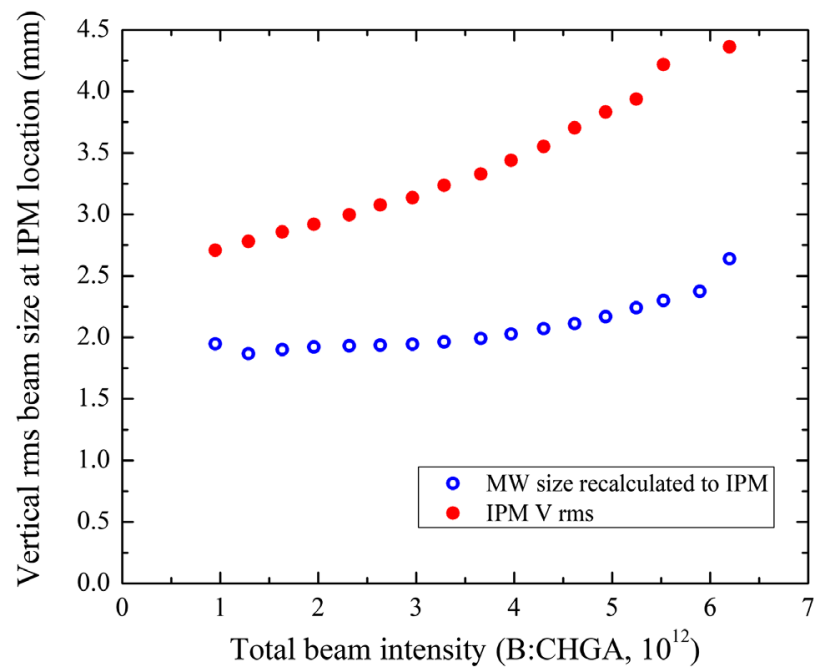

FIG. 9. Comparison of the measured rms IPM vertical beam sizes at extraction for different beam intensities with the rms sizes measured by the MWs and recalculated to the IPM location.

keep the trajectories of the secondaries parallel to the electric field.

Contrary to the MWs, the IPMs exhibit intrinsic dependence on the proton beam intensity as the proton space charge fields lead to transverse expansion of the cloud of ions on its way from its origin (in the proton beam) to the IPM detector plate-see Fig. 9. A comprehensive theory of the IPM operation is developed in [45]. The rms size of the measured profile in the IPM $\sigma_{m}$ is related to the original proton beam size $\sigma_{0}$ as

$$
\sigma_{m}=\sigma_{0} \cdot h\left(N_{p}, \sigma_{0}, D, V, d\right)
$$

where the expansion factor $h$ can be approximated as

$$
h \approx 1+F\left(\frac{2 \Gamma(1 / 4) U_{\mathrm{SC}} D d^{1 / 2}}{3 V \sigma_{0}^{3 / 2}}\right)\left(1+t_{b} / \tau_{0}\right)
$$

Here $V$ is the IPM extracting voltage (typically, $24 \mathrm{kV}$ in our case) and $D$ is its high voltage gap (103 mm), $d$ is the distance for ions to travel from the beam orbit to the IPM collection plate, the space-charge potential for the proton beam with current $I$ is $U_{\mathrm{SC}}=30[\mathrm{~V}] I / \beta_{p}$ and gamma function $\Gamma(1 / 4) \approx 3.625$. The numerical factor $F$ is equal to 1 in the case of unbunched dc proton beam with Gaussian transverse current distribution, and $F=$ $2 \sqrt{2} / \Gamma(1 / 4) \approx 0.78$ for uniform distribution with radius $a=2 \sigma_{0}$. In the case of the Booster, with modest expansion $h \leq 2$, one can neglect minor corrections due to somewhat unequal horizontal and vertical beam sizes, but should take into account the correction factor $\left(1+t_{b} / \tau_{0}\right)$ due to the bunch structure of the Booster proton current. There, $t_{b}$ is the bunch spacing (about $19 \mathrm{~ns}$ at the end of the Booster cycle) and characteristic time for an ion to leave the beam 
$\tau_{0}=\sqrt{2 M D \sigma_{0} / Z e V}$, where $M$ and $e Z$ are the ion's mass and charge, which is about 22 ns for typical IPM and beam parameters at the end of the cycle.

Also very important are intensity independent effects leading to the IPM profile smearing, such as the initial velocities of the ions, finite separation between the individual IPM charge collection strips, angular misalignment of the IPM long and narrow strips with respect to the highenergy proton beam orbit, etc. [45]. These effects are monitor specific; they add in quadrature and can be determined in comparison with the low intensity beam sizes measured by the IPM and by the MWs $\sigma_{T}^{2}=\sigma_{m, \mathrm{IPM}}^{2}-$ $\sigma_{m, \mathrm{MW}}^{2}$ at $N_{p} \rightarrow 0$. For the Booster IPMs, it was found that such instrumental smearing is $\sigma_{T}^{2}=2.8 \pm 0.1 \mathrm{~mm}^{2}$ and correspondingly modified Eq. (3) $\sigma_{\mathrm{IPM}}^{2}=\sigma_{T}^{2}+h^{2} \sigma_{\mathrm{MW}}^{2}$ describes the Booster IPM data at extraction with some $5 \%$ accuracy [46].

The original proton beam size $\sigma_{0}$ can be found from the measured and correspondingly corrected IPM value of $\sigma^{*}=\sqrt{\sigma_{m, \mathrm{IPM}}^{2}-\sigma_{T}^{2}}$ by reversing the equation $\sigma^{*}=$ $\sigma_{0}\left(1+c N_{p} / \sigma_{0}^{3 / 2}\right)$, where $c$ is deducted from Eqs. (3) and (4), if other parameters, such as $\sigma_{T}, N_{p}, d$ and the IPM voltage $V$ and gap $D$, are known. A simple practical algorithm gives better than $\pm 5 \%$ approximation over the entire range of the Booster beam intensities:

$$
\sigma_{0} \approx \frac{\sigma^{*}}{\left(1+c N_{p} / \sigma^{* 3 / 2}\right)\left(1+\alpha c^{2} N_{p}^{2} / \sigma^{* 2}\right)} .
$$

For the highest beam intensity in our studies, $N_{p}=6 \times 10^{12}$, the factor $c N_{p}=2.53 \mathrm{~mm}^{3 / 2}$ and the fitting coefficient $\alpha \approx 0.4$ [46]. Equation (5) can now be used to find the proton beam size over the entire Booster cycle, i.e., not just for the values measured at extractions. For example, the black lines in Fig. 10 represent the raw (uncorrected) rms vertical and horizontal beam sizes $\sigma_{m, \text { IPM }}$ as measured by the IPM at each of 20000 turns of the Booster acceleration cycle. The pink and green lines represent the beam sizes $\sigma^{*}$ corrected for the intensity independent smearing $\sigma_{T}$. The true proton rms beam sizes $\sigma_{0}$ reconstructed following the above algorithm [Eq. (5)] are represented by the red and blue lines. One can see that the overall beam size correction is about $10 \%-15 \%$ early in the Booster acceleration cycle when the rms beam size is about $5 \mathrm{~mm}$. At the end of the cycle, with proton energy increased from $400 \mathrm{MeV}$ to $8 \mathrm{GeV}$, the proton beam size is almost a factor of about 1.8 smaller than it appears in the IPM and accounting for the space-charge expansion $h\left(N_{p}, D, V, d\right)$ is the most important. The reconstructed beam sizes $\sigma_{0}$ at the end of the acceleration cycle match the extracted beam size measured by the MWs well.

As can be seen in Fig. 10, the horizontal rms beam size exhibits significant oscillations with twice the synchrotron
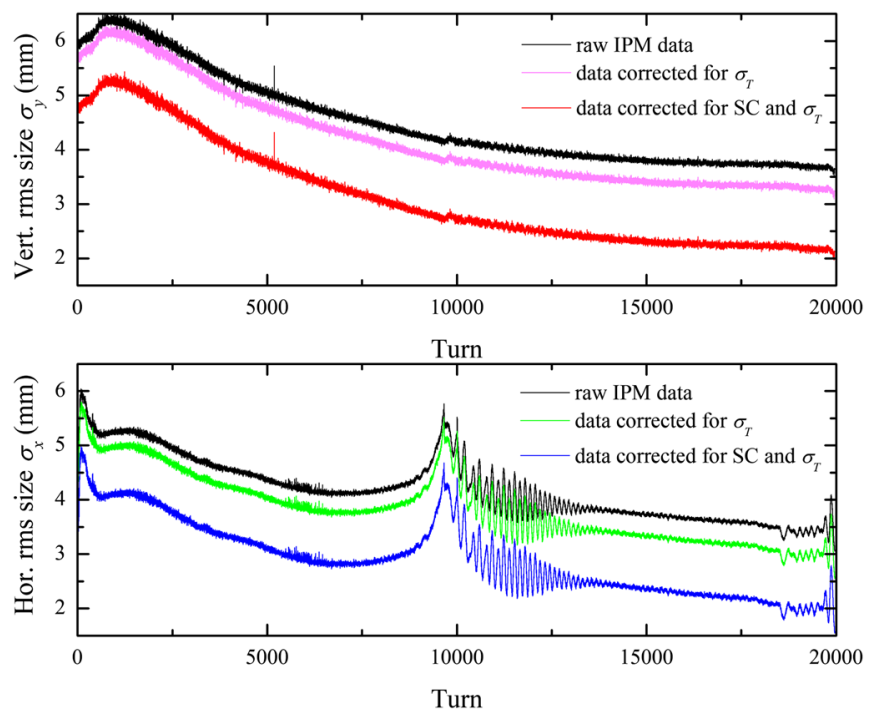

FIG. 10. An example of reconstruction of vertical (top plot) and horizontal (bottom plot) rms proton beam sizes in the $33 \mathrm{~ms} \mathrm{(20}$ 000 turns) acceleration cycle of the Fermilab $8 \mathrm{GeV}$ Booster synchrotron with the total beam intensity of $N_{p}=4.6 \times 10^{12}$ : time dependence of the original IPM data $\sigma_{m, \text { IPM }}^{2}$, the data corrected for smearing effects $\sigma^{*}$ and the same data after additional correction for the space-charge expansion $\sigma_{0}$ with parameters $D=103 \mathrm{~mm}, d=52 \mathrm{~mm}, V=24 \mathrm{kV}$-see text and Eqs. (4) and (5).

frequency after the transition. Such oscillations arise from the mismatch between longitudinal focusing of bunch fields before and after transition, and the effect gets bigger with intensity. Horizontal IPM is located at small beta function and high dispersion, so, compared to the betatron size, the dispersive contribution is large $D_{x}(\delta p / p) \geq$ $\sqrt{\varepsilon_{x} \beta_{x} /\left(\beta_{p} \gamma_{p}\right)}$ and variations in the momentum spread $(\delta p / p)$ result in about $\pm 20 \%$ oscillations in $\sigma_{x}$. The Booster is well decoupled and, consequently, the vertical

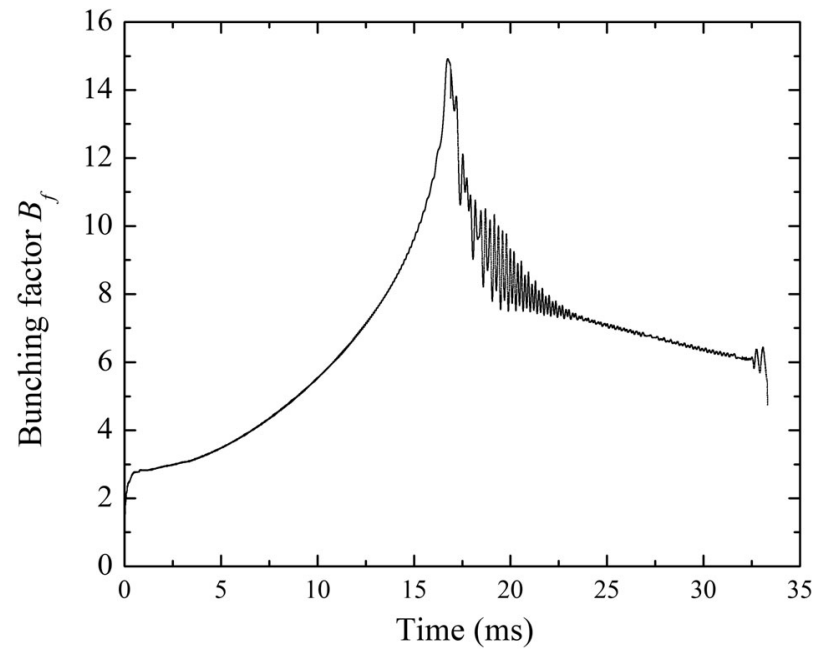

FIG. 11. Booster bunching factor over the accelerator ramp (ratio of the peak to average proton beam current). 
dispersion is small. Therefore a little perturbation observed at transition is most probably due to reaction of the IPM profile expansion factor $h$ to variation of the bunching factor $B_{f}=\sqrt{2 \pi} \beta_{p} c / f_{r f} \sigma_{s}$ - the ratio of the peak to average proton beam current-which peaks at the transition-see Fig. 11. The effect is small but becomes more pronounced at higher intensities [45].

\section{B. Beam emittance vs intensity}

The injected Linac $H^{-}$beam comes with the rms normalized transverse emittances $\varepsilon \sim 1 \pm 0.2 \pi \mathrm{mm}$ mrad [47]. Due to small injection errors, optics mismatch and multiple scattering in the foil, the initial emittance of the proton beam circulating in the Booster gets to about $\sim 1.2 \pm 0.2 \pi \mathrm{mm}$ mrad. The latter effect correlates with the total beam intensity. Indeed, in the process of multiturn charge exchange injection, each passage of the stripping foil leads to the emittance growth of

$\Delta \varepsilon_{x, y} \approx \beta_{p} \gamma_{p} \frac{\beta_{x, y}}{2} \frac{l}{X_{0}}\left(\frac{13.6 \mathrm{MeV}}{\beta_{p} p c}\right)^{2}\left[1+0.0038 \ln \left(l / X_{0}\right)\right]$.

For the Booster carbon foil thickness $l=380 \mu \mathrm{g} / \mathrm{cm}^{2}$, radiation length $X_{0}=42.7 \mathrm{~g} / \mathrm{cm}^{2}$ and momentum $p=$ $953 \mathrm{MeV} / c$ that gives $0.0032 \pi \mathrm{mm} \mathrm{mrad}$ per turn in the horizontal plane and $0.011 \pi \mathrm{mm} \mathrm{mrad}$ per turn in the vertical plane. During the injection, the beam moves across and off the foil, so the effective number of turns is about $\left(N_{\text {turns }}+29\right) / 2$ turns [48]. Given that the total circulating beam intensity $N_{p}$ scales linearly with $N_{\text {turns }}$, the estimated emittance increase at the end of injection grows with the intensity and for the nominal $N_{\text {turns }}=14$ turns injection $\Delta \varepsilon_{y, \text { foil }} \approx 0.24 \pi \mathrm{mm} \mathrm{mrad}$ and $\Delta \varepsilon_{x, \text { foil }} \approx 0.07 \pi \mathrm{mm} \mathrm{mrad}$.

Measured Booster beam emittances at extraction exhibit strong dependence on the total proton intensity $N_{p}$, as shown in Fig. 12. The MWs data taken at the nominal operational chromaticities at injection $Q_{x, y}=-16 /-4$ show strong dependence on the beam intensity and for both planes can be approximated as

$$
\varepsilon_{\text {extr }}[\pi \mathrm{mm} \mathrm{mrad}] \approx 1.7+2.1 \cdot\left(\frac{N_{p}}{7 \times 10^{12}}\right)^{4 \pm 0.3} .
$$

The emittance growth is strongly dependent on the chromaticity, too, and at the nominal intensity $N_{p}=4.5 \times$ $10^{12}$ the emittance increases from about $2.1 \pi \mathrm{mm}$ mrad to some $2.4 \pi \mathrm{mm}$ mrad (vertical) and $2.7 \pi \mathrm{mm}$ mrad (horizontal) if the operational chromaticity at the first ms after the injection is changed from $Q_{x, y}=-16 /-4$ to $-20 /-20$. The beam emittance increase with intensity and chromaticity strongly correlates with the intensity losses-see Eqs. (1) and (2) and Figs. 6 and 7.

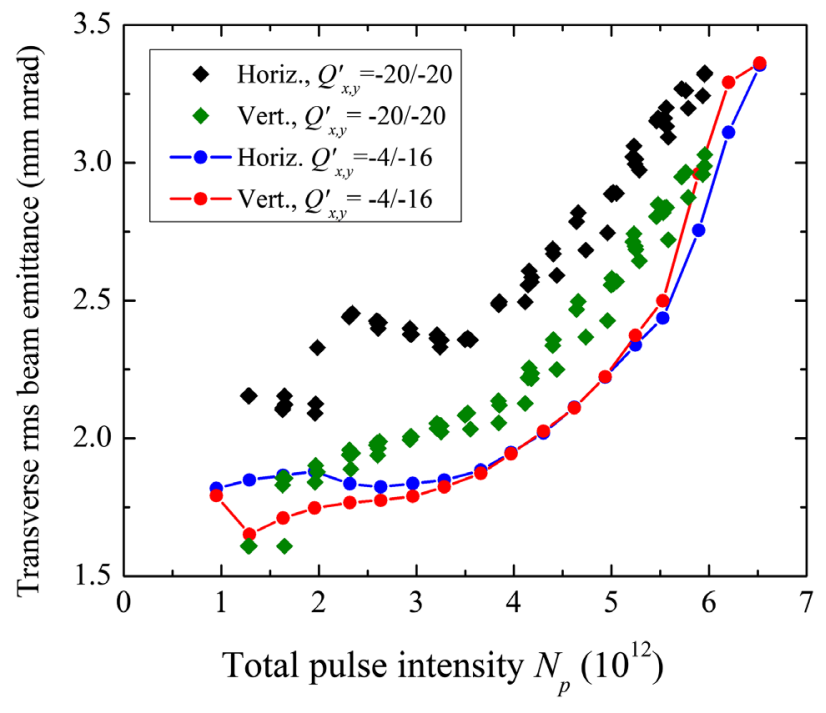

FIG. 12. Booster beam emittance measured by MWs at extraction vs the total proton intensity.

The IPM rms beam sizes $\sigma_{0, x, y}(t)$ measured over the Booster acceleration cycle from injection to extraction and properly corrected following the analysis of preceding Sec. IVA and Eq. (5) can be used for the emittance calculations $\varepsilon_{y}=\left(\beta_{p} \gamma_{p}\right) \sigma_{0, y}^{2} / \beta_{y}, \varepsilon_{x}=\left(\beta_{p} \gamma_{p}\right)\left[\sigma_{0, y}^{2}-\right.$ $\left.D_{x}^{2}(\delta p / p)^{2}\right] / \beta_{x}$, where $\delta p / p$ is the rms energy spread. Of course, all the factors are now time dependant: the relativistic factors $\beta_{p}(t)$ and $\gamma_{p}(t)$ are well known-see, e.g., Fig. 2; the beta functions $\beta_{x, y}$ at the IPM locations vary in the cycle within $\sim 10 \%$. Calculations of Booster emittance taking all these effects into account agree with the MWs data at extraction to within $10 \%$ and exhibit no emittance growth at the lowest intensities—see [46].

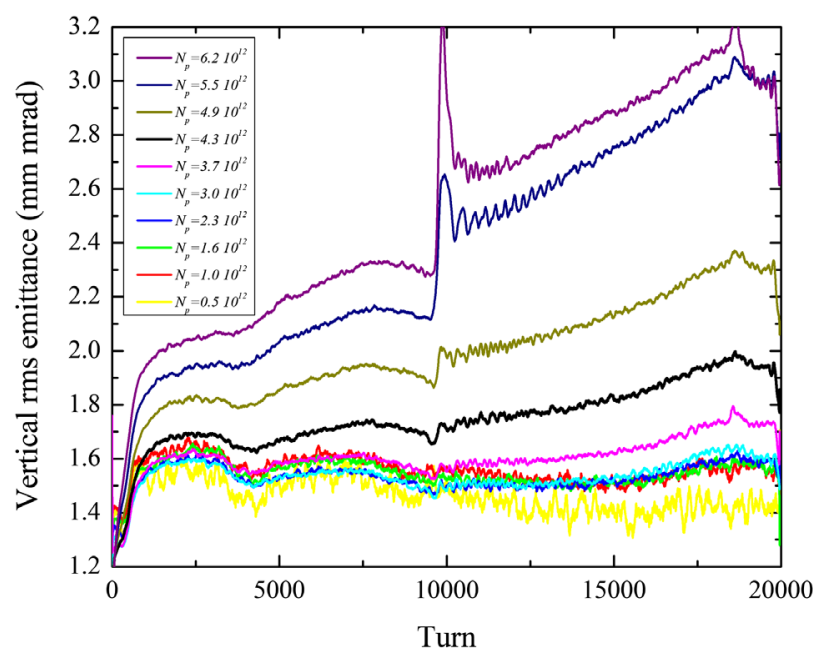

FIG. 13. Evolution of the IPM vertical emittance in the Booster cycle at different intensities $N_{p}$ from $0.5 \times 10^{12}$ (2 turns injection) to $6.2 \times 10^{12}$ (20 turns injection). All the data are smoothed by a 100 turn running window averaging. 
The resulting Booster IPM vertical beam emittance evolution over the acceleration cycle is shown in Fig. 13 for a wide range of intensities $N_{p}$ from $0.5 \times 10^{12}$ ( 2 turns injection) to $6.2 \times 10^{12}$ (20 turns). For example, the emittance $\varepsilon_{y}(t)$ at the operational intensity of $N_{p}=4.3 \times$ $10^{12}$ is shown in the black line in Fig. 13. Shortly after injection it is about $1.3 \pm 0.1 \mathrm{~mm} \mathrm{mrad}$ and evolves to $1.9 \pm 0.1 \mathrm{~mm}$ mrad at extraction in good agreement with the MWs emittance data shown in Fig. 12. In general, one can see that up to about $N_{p}=3.7 \times 10^{12}$ (12 turns injection, pink line) the emittance is not growing much in the cycle and is about $1.4-1.6 \mathrm{~mm}$ mrad. Above that intensity the emittance evolution exhibits several features: (i) fast growth over the first 2000-3000 turns; (ii) steady growth for the rest of the cycle; (iii) spikes at the time of transition and minor oscillations afterwards; and (iv) 5\%$10 \%$ variations at the end of the cycle. The last two effects are presumably instrumental. Significant variation of the bunching factor at the transition shown in Fig. 11 does affect the IPM profile expansion $h$; see Eq. (4). At the end of the acceleration cycle, the proton beam position in the IPMs varies over the last 2000 turns by as much as $6 \mathrm{~mm}$ in the horizontal plane, thus affecting the IPM profile expansion factor $h_{y}$ in the vertical plane that scales with the distance from the beam orbit to the IPM collection plate as $d_{x, y}^{1 / 2}$-see Eq. (4), while bunch rotation in longitudinal phase-space prior to the extraction at the very last hundreds of turns results in a smaller momentum spread and longer bunches-again, leading to minor variation of the IPM expansion factor $h$.

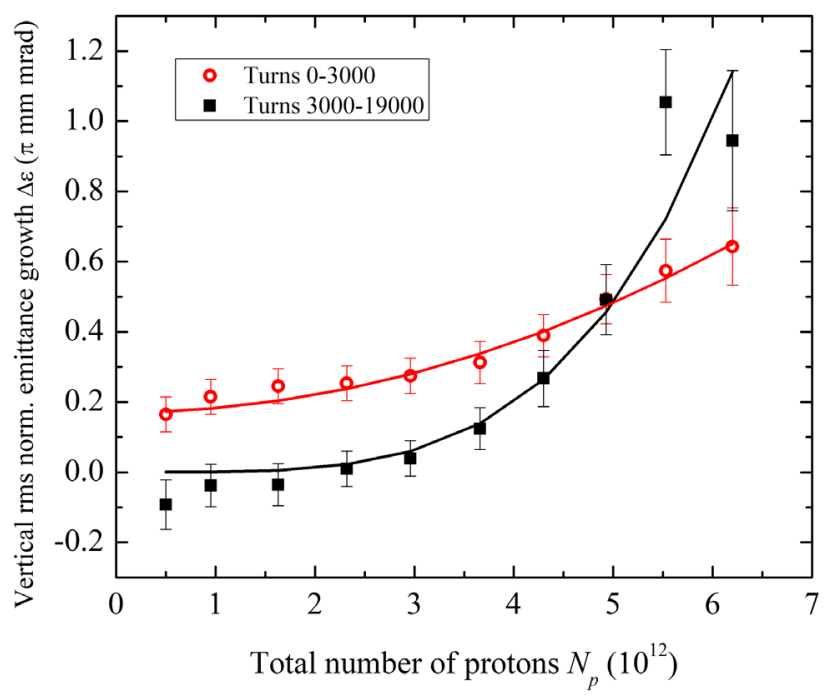

FIG. 14. Vertical rms emittance growth vs $N_{p}$ : (red circles) over the first 3000 turns; (black squares) from 3000 to 19000 turns. The data points are calculated for the IPM emittance values averaged over 500 turns $0-500,3000-3500,19000-19500$. The error bars indicate estimated statistical uncertainty. Red and black solid line are for the approximations Eqs. (8) and (9), respectively.
The most dominant are the first two effects. Figure 14 shows how they depend on the beam intensity. The fast rms vertical emittance growth over the first 3000 turns is most probably due to record strong proton space-charge effects (see below) and scales approximately as

$\Delta \varepsilon_{y, 3000}[\pi \mathrm{mm} \mathrm{mrad}] \approx 0.17+0.61 \cdot\left(\frac{N_{p}}{7 \times 10^{12}}\right)^{2}$.

Slow emittance increase is roughly linear in time over the next 16000 turns and gets as big as $1 \mathrm{~mm} \mathrm{mrad}$, or $30 \%$ of the emittance, at $N_{p}=6.2 \times 10^{12}$ and can be approximated as

$$
\Delta \varepsilon_{y, 3000-19000}[\pi \mathrm{mm} \mathrm{mrad}] \approx 1.85 \cdot\left(\frac{N_{p}}{7 \times 10^{12}}\right)^{4} .
$$

The nature of that effect might be related to the multipacting of electrons in the beam and/or beam-induced vacuum activity, but that still needs to be confirmed [49].

\section{Space-charge tune shift}

Space-charge tuneshift parameter $\Delta Q_{\mathrm{SC}}$ is a commonly used figure of merit for beam dynamics. It is equal to [4]

$$
\Delta Q_{\mathrm{SC}}=\frac{N_{p} r_{p} B_{f}}{4 \pi \varepsilon \beta_{p} \gamma_{p}^{2}} R
$$

where $N_{p}$ is the total intensity, assuming that the bunches fill all rf buckets, $r_{p}$ is the classical proton radius, $B_{f}$ is the bunching factor (the ratio of the peak to average bunch current), $\varepsilon$ is the normalized rms beam emittance, and $\beta_{p}$ and $\gamma_{p}$ are relativistic Lorentz factors. Factor $R \lesssim 1$ accounts for unequal average beam size ratio around the ring and, e.g., for the vertical plane it is equal to $\left\langle 2 /\left(1+\sigma_{x} / \sigma_{y}\right)\right\rangle$. The tuneshift is negative, but we omit the minus sign for simplicity. In operational circular rapid cycling accelerators, the space-charge parameter usually does not exceed $0.3-0.5$ to avoid beam losses [4,50,51].

Figure 15 shows the vertical SC tuneshift parameter $\Delta Q_{\mathrm{SC}}(t)$ calculated for the Fermilab Booster acceleration cycle on a base of measured $N_{p}(t), \varepsilon_{y}(t), B_{f}(t)$ and known $\beta_{p}(t)$ and $\gamma_{p}(t)$. One can see that the calculated spacecharge parameter quickly grows after the injection due to fast bunching early in the acceleration cycle, then falls down due to acceleration and emittance increase, and exhibits some temporary increase at the transition before ending at $\sim 0.01$ prior to extraction. The maximum Booster tuneshift parameter peaks at about $1 \mathrm{~ms}$ after injection to $\Delta Q_{\mathrm{SC}} \simeq 0.65$ and stays above 0.3 until about $6 \mathrm{~ms}(\sim 3000$ turns). Naturally, the corresponding incoherent spacecharge tune spread does not easily fit the available tune space between most dangerous resonances, such as 


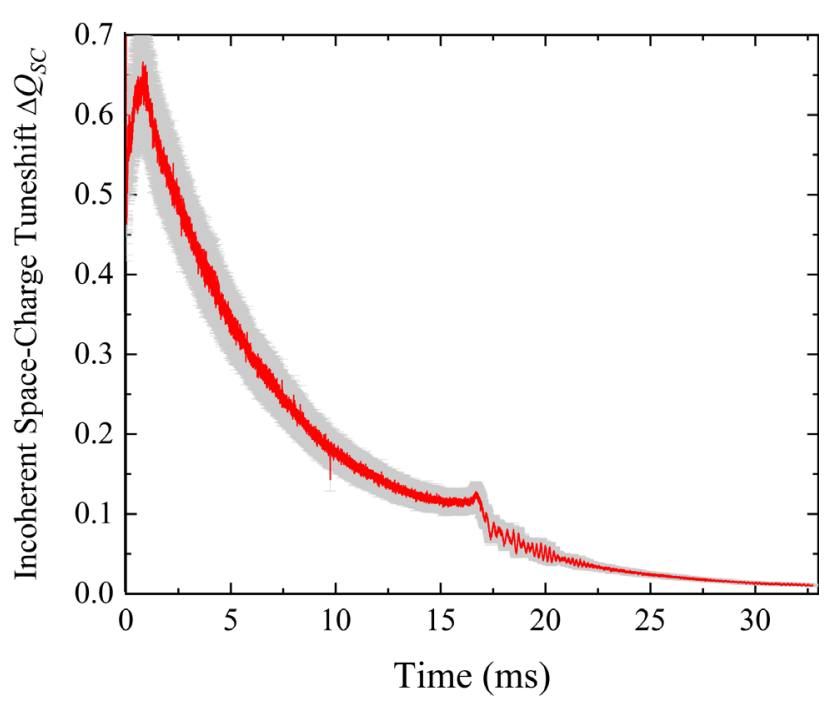

FIG. 15. Calculated vertical space-charge tuneshift parameter for the Booster cycle with $N_{p}=4.6 \times 10^{12}$. (The shaded area indicates $10 \%$ uncertainty, mostly due to the IPM emittance calculations.)

half-integer ones, and that results in strong resonant excitation of the proton dynamics and eventual particle losses at the machine aperture. At the highest beam intensity studied, $N_{p}=6.2 \times 10^{12}$, the maximum spacecharge tuneshift parameter $\Delta Q_{\mathrm{SC}}$ peaks at $\simeq 0.75$.

\section{SUMMARY, DISCUSSION}

Detrimental effects in high-intensity, high-brightness beams impose serious limitations on the performance of proton and ion RCSs [4,51] and were the subject of operational optimizations and dedicated experimental studies at the CERN PS and PS Booster accelerators [52-57], BNL's AGS and its booster [58], Fermilab Booster [15,59], ISIS at RAL [60,61], SIS18 at GSI [62], J-PARC RCS and main ring [63-65], CSNS/RCS in China [66], and other machines. Beam losses and emittance growth are usually a result of a complex interpla of factors which might include space-charge effects and coherent instabilities (dipole and higher order ones) in transverse and longitudinal planes, machine working points and chromaticities, focusing lattice type, symmetry, nonlinearities and errors, type and locations of the rf accelerating systems, physical aperture and collimation system parameters, crossing transition energy methods and speed of acceleration, etc. Experimental measurements provide critical data for benchmarking and further in-depth theoretical studies and numerical simulations, see, e.g., Refs. [12,67-82].

The phenomena of the Fermilab Booster beam intensity losses, transverse emittance growth and longitudinal beam excitation at the transition are found to be very much intensity dependent and strongly interconnected. Our experimental studies indicate that the losses that occur early in the cycle are most probably due to the space-charge driven vertical beam size expansion that leads to the flux of protons on the collimator-limited machine aperture with characteristic acceptance $A_{y} \simeq 20-25[\pi \mathrm{mm} \mathrm{mrad}]$ [46]. Both the fractional beam loss [Eqs. (1) and (2)] and the emittance growth [Eq. (8)] highly nonlinearly depend on the total circulating beam intensity $N_{p}$.

Beam losses at and after the transition crossing, while small under conditions of optimal operational tune up to the nominal intensity $N_{p}=4.6 \times 10^{12}$, exhibit a thresholdlike behavior above $6 \times 10^{12}$. IPM measurements indicate small vertical beam size at the transition while the horizontal size, dominated by the dispersive contribution $D_{x}(\delta p / p)$, becomes very large, so it is natural to assume that the proton losses at the transition end up at the horizontal aperture.

Booster is a lynch pin of the Fermilab proton accelerator complex, and its transmission efficiency has been the subject of continuous monitoring and several studies for almost four decades-see, e.g. [59,83,84]. Naturally, the Booster performance at present significantly exceeds that of the past-compare current transmission of $\sim 95 \%$ at $N_{p}=(4.5-5) \times 10^{12}$ with that of $\sim(70-75) \%$ at $N_{p}=$ $(3.5-4) \times 10^{12}$ in $1980 \mathrm{~s}$ and $1990 \mathrm{~s}$. What makes this work distinct is that for the first time we have performed a simultaneous comprehensive analysis of the proton loss dependence on the total beam intensity, machine tunes and chromaticities, and, also, performed a cross calibration of the toroid intensity monitor and the beam loss monitors.

Nonlinear dependence of the extracted Booster beam emittance on $N_{p}$ was reported in many previous measurements with MultiWires profile monitors-see, e.g., $[83,85,86]$. The Booster ionization profile monitors operating in the ion collection mode without external magnetic field are known to be extremely valuable tools for fast beam size diagnostics of the circulating beam during acceleration $[44,87,88]$. At the same time, strong space-charge forces of the high-intensity proton beam might lead to significant, by a factor of 2 or more, expansion of the rms beam size reported by the IPMs compared to the original proton beam size [45]. To be certain that we properly account for that and other, intensity-independent, effects, we have performed a systematic calibration of the IPM with the MultiWires measurements as described in Sec. IVA. As a result, we have achieved $O(10) \%$ accuracy in the beam emittance reconstruction using the measured beam intensity $N_{p}$ and IPM rms beam sizes, experimentally determined intensity-independent instrumental dispersion $\sigma_{T}$, and the IPM parameters such as extracting electric field $E_{\text {ext }}=V_{0} / D$ and the distance $d$ from the beam orbit to the IPM detector.

Our data indicate the existence of two phenomena leading to the proton beam emittance growth: (i) the space-charge driven expansion over the first few thousand turns; and (ii) steady emittance increase over the rest of the acceleration cycle. Both effects grow faster than linearly with the proton beam intensity, resulting in the final 
(extracted) beam emittance having a significant component $\propto N_{p}^{4}$ - see Eq. (7), which also depends on the machine chromaticity at the injection energy. An attempt at a similar analysis of the Booster IPM profiles has been undertaken in [15]. It was based on the phenomenological approximations for the IPM profile space-charge induced expansion effects developed in [88], and reported space-charge driven emittance growth early in the cycle was found to be (a) significantly (3-4 times) bigger than reported here; and (b) scaling approximately linear with intensity $\propto N_{p}$. These observations also show improbably large normalized rms emittances of high-intensity beams prior to extraction $\sim 10[\pi \mathrm{mm} \mathrm{mrad}]$, thus directly contradicting our MultiWire measurements and what we know about the Booster acceptance; this makes us suspect a systematic error in the IPM data analysis. Notably, the steady emittance increase during the acceleration cycle was observed, too, though again, it was more than twice of what we report here in Eq. (9).

Our experimental studies of the Booster losses and emittance evolution are of great importance to predict the machine operational conditions in the era of the upcoming new $800 \mathrm{MeV}$ injector (PIP-II) and have to be continued. These studies and the future operation of the Booster with up to $50 \%$ higher beam intensities would greatly benefit from improved accuracy of the fast beam intensity diagnostics and of the IPM emittance analysis. The losses at transition are believed to result from complicated 3D dynamics, including transverse and longitudinal coherent instabilities. Future investigations of the dependence on the rf voltage, chromaticities, tunes and other machine parameters will help to better identify the transition loss origin. ESME [89] and BLonD [90] simulations are also under way to study the impact of $\gamma_{t}$ jumps on transition in the PIP-II era.

The strong intensity dependence of the space-charge induced losses in the first few thousands of turns after the injection is also of concern. Indeed, let us consider an RCS accelerator, like the Fermilab Booster, operationally limited by the uncontrolled radiation level in accelerator enclosures at $W=f_{0} \int E_{k} d N_{p}$ (here $f_{0}$ is the cycle rate and $E_{k}$ is the kinetic energy of the lost particle) - typically it is about $1 \mathrm{~W}$ per meter of machine circumference. Under such a limit, the tolerable fractional beam intensity loss is

$$
\frac{\Delta N_{p}}{N_{p}}=\frac{W}{(1-\eta) N_{p} E_{k} f_{0}},
$$

where $\eta$ is the efficiency of the collimation system that directs the losses into dedicated beam absorbers or dumps. Obviously, the losses should get smaller with the increase of beam intensity, energy and power. Instead, many beam physics phenomena, such as, e.g., repelling forces of the proton beam's own space charge, lead to increase of beam sizes and particle losses at higher beam intensities. Let us, following Eq. (1), consider the case when the space-charge induced losses grow with the space-charge tuneshift $\Delta Q_{\mathrm{SC}}$ as

$$
\frac{\Delta N_{p}}{N_{p}} \propto \alpha \Delta Q_{\mathrm{SC}}^{\kappa}
$$

where $\alpha$ is a machine dependent constant and the exponent $\kappa$ is about 3 . Though such scaling is empirical and perhaps specific to the Booster operational conditions until further proof of its underlying beam dynamics mechanisms via theoretical analysis and numerical modeling, it can be used for preliminary evaluation of the relative importance of the main operationally available opportunities for the machine performance improvements. From Eqs. (11) and (12) one gets the maximum operational intensity within the loss power limit $W$ :

$$
N_{p}^{\max } \propto\left(\frac{W}{1-\eta}\right)^{\frac{1}{\kappa+1}}\left(\frac{\varepsilon}{B_{f}}\right)^{\frac{\kappa}{\kappa+1}} \frac{\gamma_{p}^{\frac{2 \kappa-1 / 2}{k+1}}}{\left(\alpha f_{0}\right)^{\frac{1}{k+1}}} .
$$

From that, one can see that there are several paths to the increase of the maximum intensity [18], but none leads to direct one-to-one increase of $N_{p}^{\max }$. Assuming $\kappa=3$, Eq. (13) anticipates that a threefold improvement in collimation system efficiency (e.g., from $\eta=0.55$ to 0.85 ) [91] leads to a $3^{1 / 4}$ increase in $N_{p}^{\max }$, or $32 \%$. For the twofold increase in Booster injection energy $E_{k}$ from 400 to $800 \mathrm{MeV}$ with a new PIP-II linac [21], Eq. (13) predicts that one should be able to safely increase $N_{p}^{\max }$ by $41 \%$ from the current operational value, except the increase in cycle rate $f_{0}$ from 15 to $20 \mathrm{~Hz}$ under the same plan will cut the expected benefit to just $31 \%$. Equation (13) indicates that the two aforementioned improvements combined should safely allow the PIP-II project goal of $44 \%$ Booster intensity increase from $4.5 \times 10^{12}$ to $6.5 \times 10^{12}$ protons per pulse.

More broadly, Eq. (13) evaluates strategies for improving intensity in other high-intensity rings. Flattening the longitudinal bunch current profile, e.g., by using additional second or third harmonics rf systems, leads to reduction of the bunching factor, and the factor of 2 smaller $B_{f}$ could lead to 1.68 times higher maximum intensity. Acceleration of twice larger emittance beams would give about the same effect, but it is usually not possible within the available machine aperture of existing machines. At ultimate intensities, significant promise in loss reduction lies in improved beam dynamics that would make $\alpha$ and $\kappa$ smaller, for example by injection painting to make the space-charge forces more uniform, by compensation of the most detrimental resonant driving terms (including enforcement of perfect periodicity in machine focusing optics), by spacecharge compensation using electron lenses [92], or by implementation of the nonlinear integrable optics [93]. 
For the latter two topics in particular, an R\&D program is under way at the Fermilab IOTA facility [94].

Our studies of the beam intensity dependent effects in the Fermilab Booster provide a rich experimental dataset for benchmarking numerical simulation codes used to explain observed phenomena in existing high-intensity, high brightness proton accelerators at Fermilab and to predict operational performance of their upgrades and future rapid cycling synchrotrons.

\section{ACKNOWLEDGMENTS}

We would like to thank C. Y. Tan, C. Bhat, Yu. Alexahin, A. Burov, W. Pellico, R. Thurman-Keup, S. Nagaitsev and $R$. Baartman for numerous discussions on the topics of this study and S. Chaurize, V. Kapin and K. Triplett for their invaluable help with experimental Booster beam studies. In addition, the Summer 2019 Booster beam study campaign involved N. Eddy, C. Jensen, J. Larson, and H. Pfeffer of Fermilab, H. Bartosik, N. Biancacci, M. Carla, A. Saa Hernandez, A. Huschauer, F. Schmidt of CERN, D. Bruhwiler, J. Edelen of the Radiasoft SBIR company and V. Kornilov of GSI. We greatly appreciate their fruitful cooperation and the spirit of international beam physics collaboration. We also would like to thank V. Higgins for carefully reading through the manuscript and helpful feedback. This manuscript has been authored by Fermi Research Alliance, LLC under Contract No. DE-AC0207CH11359 with the U.S. Department of Energy, Office of Science, Office of High Energy Physics.

[1] V. Shiltsev, On performance of high energy particle colliders and other complex scientific systems, Mod. Phys. Lett. A 26, 761 (2011).

[2] V. Shiltsev, Particle beams behind physics discoveries, Phys. Today 73, 32 (2020).

[3] V. Shiltsev, Superbeams and neutrino factories - two paths to intense accelerator-based neutrino beams, Mod. Phys. Lett. A, 2030005 (2020).

[4] J. Wei, Synchrotrons and accumulators for high-intensity proton beams, Rev. Mod. Phys. 75, 1383 (2003).

[5] R. Zwaska et al., Multi-MW targets for next-generation accelerators, in Proceedings of the International Particle Accelerator Conference (IPAC'18), Vancouver, BC, Canada (JACoW, Geneva, Switzerland, 2018).

[6] N. Simos, P. Hurh, E. Dooryhee, L. Snead, D. Sprouster, Z. Zhong, H. Zhong, S. Ghose, Z. Kotsina, K. Ammigan et al., $120 \mathrm{GeV}$ neutrino physics graphite target damage assessment using electron microscopy and high-energy $\mathrm{x}$ ray diffraction, Phys. Rev. Accel. Beams 22, 041001 (2019).

[7] E. L. Sola, M. Calviani, O. Aberle, C. Ahdida, P. Avigni, M. Battistin, L. Bianchi, S. Burger, J. B. Descarrega, J. C. Espadanal et al., Beam impact tests of a prototype target for the beam dump facility at CERN: Experimental setup and preliminary analysis of the online results, Phys. Rev. Accel. Beams 22, 123001 (2019).

[8] A. W. Chao, Physics of Collective Beam Instabilities in High Energy Accelerators (Wiley, New York, 1993).

[9] N. Diskansky and D. Pestrikov, The Physics of Intense Beams and Storage Rings (AIP Press, Woodbury, NY, USA, 1997).

[10] K.-Y. Ng, Physics of Intensity Dependent Beam Instabilities (World Scientific, Singapore, 2006).

[11] M. Reiser, Theory and Design of Charged Particle Beams (John Wiley and Sons, New York, 2008).

[12] I. Hofmann, Space Charge Physics for Particle Accelerators (Springer, New York, 2017).

[13] M. Minty and F. Zimmermann, Measurement and Control of Charged Particle Beams (Springer-Verlag, Berlin-Heidelberg-New York, 2003).

[14] P. Strehl, Beam Instrumentation and Diagnostics (Springer, New York, 2006), Vol. 120.

[15] X. Huang, S. Lee, K. Ng, and Y. Su, Emittance measurement and modeling for the Fermilab Booster, Phys. Rev. ST Accel. Beams 9, 014202 (2006).

[16] K. Wittenburg, Specific instrumentation and diagnostics for high-intensity hadron beams, arXiv:1303.6767.

[17] J. Eldred, Physics studies for high intensity proton beams at the Fermilab Booster, in Proceedings of NAPAC'19, Lansing, MI, 2019 (JACoW, Geneva, Switzerland, 2019).

[18] V. Shiltsev, Fermilab proton accelerator complex status and improvement plans, Mod. Phys. Lett. A 32, 1730012 (2017).

[19] M. Convery, M. Lindgren, S. Nagaitsev, and V. Shiltsev, Fermilab accelerator complex: Status and improvement plans, Technical Report No. FERMILAB-TM-2693, Fermilab, 2018.

[20] R. Acciarri et al., Long-baseline neutrino facility (LBNF) and deep underground neutrino experiment (DUNE) conceptual design report volume 1: The LBNF and DUNE projects, arXiv:1601.05471.

[21] V. Lebedev et al., The PIP-II conceptual design report, Report No. FERMILAB-TM-2649-AD-APC, Fermilab, Batavia, 2017.

[22] J. Eldred, V. Lebedev, and A. Valishev, Rapid-cycling synchrotron for multi-megawatt proton facility at Fermilab, J. Instrum. 14, P07021 (2019).

[23] J. Eldred et al., Versatile multi-MW proton facility with synchrotron upgrade of Fermilab proton complex, in Snowmass 2021 Letters of Interest (APS DPF, 2020), https://www.snowmass21.org/docs/files/summaries/AF/ SNOWMASS21-AF2_AF0-NF0_NF9_Jeffrey_Eldred092.pdf.

[24] E. Hubbard et al., Booster synchrotron, Technical Report No. FERMILAB-TM-405, Fermilab, 1973.

[25] Booster rookie book, https://operations.fnal.gov/ rookie_books/Booster_V4.1.pdf.

[26] J. Eldred, Slip-stacking dynamics for high-power proton beams at Fermilab, Ph.D. thesis, University of Indiana [Technical Report No. FERMILAB-THESIS-2015-31, 2015].

[27] K. Seiya, C. Bhat, D. Johnson, V. Kapin, W. Pellico, C.-Y. Tan, and R. Tesarek, Beam studies for the proton improvement plan (PIP)—Reducing beam loss at the Fermilab Booster, arXiv:1511.01467. 
[28] G. Budker and G. Dimov, On the charge exchange injection of protons into ring accelerators, Transactions of the International Conference on Accelerators, Dubna, 1963 (Atomizdat, Moscow, 1964), p. 993.

[29] C. Hojvat, C. Ankenbrandt, B. Brown, D. Cosgrove, J. Garvey, R. Johnson, M. Joy, J. Lackey, K. Meisner, T. Schmitz et al., The multiturn charge exchange injection system for the Fermilab Booster accelerator, IEEE Trans. Nucl. Sci. 26, 3149 (1979).

[30] C. Bhat, A new beam injection scheme for the Fermilab Booster, arXiv:1504.07174.

[31] C. Bhat, R\&D on beam injection and bunching schemes in the Fermilab Booster, arXiv:1704.08157.

[32] A. Macridin, P. Spentzouris, J. Amundson, L. Spentzouris, and D. McCarron, Coupling impedance and wake functions for laminated structures with an application to the Fermilab Booster, Phys. Rev. ST Accel. Beams 14, 061003 (2011).

[33] A. Macridin, J. Amundson, P. Spentzouris, V. Lebedev, and T. Zolkin, Transverse impedance and transverse instabilities in Fermilab Booster, Technical Report No. FERMILAB-CONF-13-431, Fermilab, 2013.

[34] A. Valishev, Y. Alexahin, and V. Lebedev, Suppression of half-integer resonance in FNAL Booster and space charge losses at injection, in Proceedings of HB2016, Malm, Sweden (JACoW, Geneva, Switzerland, 2016), pp. 164168.

[35] X. Yang, C. M. Ankenbrandt, J. MacLachlan, and V. A. Lebedev, A proposed transition scheme for the longitudinal emittance control in the Fermilab Booster, Technical Report No. FERMILAB-FN-0772, Fermilab, 2005.

[36] V. Lebedev, J.-F. Ostiguy, and C. Bhat, Beam acceleration and transition crossing in the Fermilab Booster, in Proceedings of HB2016 (Malm, Sweden) (JACoW, Geneva, Switzerland, 2016), pp. 160-163.

[37] J.-F. Ostiguy, C. Bhat, and V. Lebedev, Modeling longitudinal dynamics in the Fermilab Booster synchrotron, in Proceedings of IPAC2016, Busan, Korea (JACoW, 2016), pp. 873-876.

[38] X. Yang, A. Drozhdin, and W. Pellico, Momentum spread reduction at beam extraction from the Fermilab Booster at slip stacking injection to the Main Injector, in Proceedings of the 22nd Particle Accelerator Conference, PAC-2007, Albuquerque, NM (IEEE, New York, 2007), pp. 17331735 .

[39] B. Hendricks, ACNET: The undiscovered control system, Report No. Beams-doc-7037-v2, 2019, Fermilab internal note.

[40] V. Shiltsev, J. Eldred, V. Lebedev, and K. Seiya, Studies of beam intensity effects in the Fermilab Booster synchrotron. Part I: Introduction; Tune and chromaticity scans of beam losses, Technical Report No. FERMILAB-TM-2740, Fermilab, 2020.

[41] D. Johnson, T. Johnson, C. Bhat, S. Chaurize, K. Duel, P. Karns, W. Pellico, B. Schupbach, K. Seiya, and D. Slimmer, MEBT laser notcher (chopper) for Booster loss reduction, in ICFA ABDW on High-Intensity and HighBrightness Hadron Beams, Daejeon, Korea, 2018 (JACOW, Geneva, Switzerland, 2018), pp. 416-421.
[42] A. Burov and V. Lebedev, Impedances of laminated vacuum chambers, arXiv:1209.2996.

[43] V. Shiltsev, Y. Alexahin, V. Lebedev, P. Lebrun, R. Moore, T. Sen, A. Tollestrup, A. Valishev, and X. Zhang, Beambeam effects in the Tevatron, Phys. Rev. ST Accel. Beams 8, 101001 (2005).

[44] J. Zagel, A. Jansson, T. Meyer, D. K. Morris, D. Slimmer, T. Sullivan, and M. Yang, Operational use of ionization profile monitors at Fermilab, in Proceedings of the 14th Beam Instrumentation Workshop, Santa Fe, NM (LANL, Los Alamos, 2010).

[45] V. Shiltsev, Space-charge effects in ionization beam profile monitors, Nucl. Instrum. Methods Phys. Res., Sect. A 986, 164744 (2020).

[46] V. Shiltsev, J. Eldred, V. Lebedev, and K. Seiya, Studies of beam intensity effects in the Fermilab Booster synchrotron. Part II: Beam emittance evolution, Technical Report No. FERMILAB-TM-2741, Fermilab, 2020.

[47] C. Bhat, B. Chase, S. Chaurize, F. Garcia, K. Seiya, W. Pellico, T. Sullivan, and A. K. Triplett, Energy spread of the proton beam in the Fermilab Booster at its injection energy, arXiv:1504.07195.

[48] J. Eldred, C. Bhat, S. Chaurize, V. Lebedev, S. Nagaitsev, K. Seiya, C. Tan, and R. Tesarek, Foil scattering model for Fermilab Booster, arXiv:1912.02896.

[49] J. Eldred, Preliminary double-notch Booster ecloud study, Report No. Beams-doc-8910, 2020, Fermilab internal note.

[50] W. Chou, in ICFA Beam Dynamics Newsletter No. 20 (1999), Chap. 4.14, https://icfa-usa.jlab.org/archive/ newsletter/icfa_bd_nl_20.pdf.

[51] J. Tang, Rapid cycling synchrotrons and accumulator rings for high-intensity hadron beams, Rev. Accel. Sci. Techol. 06, 143 (2013).

[52] J. Delahaye, G. Gelato, L. Magnani, G. Nassibian, F. Pedersen, K. Reich, K. Schindl, and H. Schonauer, Shaping of proton distribution for raising the space-charge of the CERN PS Booster, in the 11th International Conference on High-Energy Accelerators (Springer, New York, 1980), pp. 299-304.

[53] G. Franchetti, I. Hofmann, M. Giovannozzi, M. Martini, and E. Metral, Space charge and octupole driven resonance trapping observed at the CERN Proton Synchrotron, Phys. Rev. ST Accel. Beams 6, 124201 (2003).

[54] M. Chanel, Space charge measurements at the PSB, Technical Report No. AB-Note-2008-006 ABP, CERN, 2008.

[55] S. Dawson, C. Williamson, G. Rumolo, and B. Mikulec, Measurements of emittance and tune spread at linac2 and linac4 injection energies for the Proton Synchrotron Booster (PSB), Technical Report No. ATS/Note/2012/ 013 PERF, CERN, 2012.

[56] F. Asvesta, H. Bartosik, S. Gilardoni, A. Huschauer, S. Machida, Y. Papaphilippou, and R. Wasef, Identification and characterization of high order incoherent space charge driven structure resonances in the CERN Proton Synchrotron, Phys. Rev. Accel. Beams 23, 091001 (2020).

[57] H. Bartosik, F. Asvesta, A. Huschauer, Y. Papaphilippou, and F. Schmidt, Space charge induced losses in the CERN injector complex, J. Instrum. 15, P07021 (2020). 
[58] L. Ahrens, J. Alessi, M. Blaskiewicz, J. Brennan, K. Brown, C. Gardner, J. Glenn, T. Roser, K. Smith, W. VanAsselt et al., High intensity performance of the Brookhaven AGS, in the 1999 Particle Accelerator Conference (IEEE PAC'99), New York (JACOW, Geneva, Switzerland, 1999), pp. 614-616.

[59] M. Popovic and C. Ankenbrandt, Performance and measurements of the Fermilab Booster, in AIP Conf. Proc. No. 448 (American Institute of Physics, New York, 1998), pp. $128-134$.

[60] G. Rees and C. Prior, Image effects on crossing an integer resonance, Part. Accel. 48, 251 (1995).

[61] C. Warsop, D. Adams, I. Gardner, B. Jones, R. Mathieson, S. Payne, B. Pine, C. Prior, G. Rees, A. Seville et al., High intensity studies on the ISIS synchrotron, including key factors for upgrades and the effects of half integer resonance, in Proceedings of HB2010, Morschach, Switzerland (JACoW, Geneva, Switzerland, 2010), pp. 619-623.

[62] G. Franchetti, O. Chorniy, I. Hofmann, W. Bayer, F. Becker, P. Forck, T. Giacomini, M. Kirk, T. Mohite, C. Omet et al., Experiment on space charge driven nonlinear resonance crossing in an ion synchrotron, Phys. Rev. ST Accel. Beams 13, 114203 (2010).

[63] A. Molodozhentsev, M. Tomizawa, and T. Koseki, Space charge effects for JPARC main ring, in Proceedings of the 22nd Particle Accelerator Conference, PAC-2007, Albuquerque, NM (Ref. [38]), pp. 3315-3317.

[64] K. Ohmi, H. Harada, S. Igarashi, and Y. Sato, Study for space charge effect in tune space at J-PARC MR, in the 5 th International Particle Accelerator Conference (IPAC'14), Dresden, Germany, 2014 (JACOW, Geneva, Switzerland, 2014), pp. 2100-2102.

[65] H. Hotchi, H. Harada, N. Hayashi, S. Kato, M. Kinsho, K. Okabe, P. Saha, Y. Shobuda, F. Tamura, N. Tani et al., Achievement of a low-loss 1-MW beam operation in the 3$\mathrm{GeV}$ rapid cycling synchrotron of the Japan Proton Accelerator Research Complex, Phys. Rev. Accel. Beams 20, 060402 (2017).

[66] S.-Y. Xu and S. Wang, Study on space charge effects of the CSNS/RCS, Chin. Phys. C 35, 1152 (2011).

[67] F. J. Sacherer, Transverse space-charge effects in circular accelerators, Ph.D. thesis, UC Berkeley [Lawrence Radiation Laboratory Technical Report No. UCRL-18454, 1968.

[68] G. Parzen, Space charge limits in proton synchrotrons, Nucl. Instrum. Methods Phys. Res., Sect. A 281, 413 (1989).

[69] S. Machida, Space-charge effects in low-energy proton synchrotrons, Nucl. Instrum. Methods Phys. Res., Sect. A 309, 43 (1991).

[70] R. Baartman, Betatron resonances with space charge, AIP Conf. Proc. 448, 56 (1998).

[71] R. Baartman, A proof due to Frank Sacherer, Technical Report No. TRI-BN-02-01, TRIUMF, 2002.

[72] S. Lee, G. Franchetti, I. Hofmann, F. Wang, and L. Yang, Emittance growth mechanisms for space-charge dominated beams in fixed field alternating gradient and proton driver rings, New J. Phys. 8, 291 (2006).
[73] M. Venturini and R. L. Gluckstern, Resonance analysis for a space charge dominated beam in a circular lattice, Phys. Rev. ST Accel. Beams 3, 034203 (2000).

[74] D. Adams, I. Gardner, B. Jones, A. Seville, H. Smith, B. Pine, C. Warsop, and R. Williamson, Beam loss studies of the ISIS synchrotron using Orbit, in the 3rd International Particle Accelerator Conference (IPAC'12), New Orleans, Louisiana, 2012 (JACOW, Geneva, Switzerland, 2012), pp. 3942-3944.

[75] A. Macridin, A. Burov, E. Stern, J. Amundson, and P. Spentzouris, Simulation of transverse modes with their intrinsic Landau damping for bunched beams in the presence of space charge, Phys. Rev. ST Accel. Beams 18, 074401 (2015).

[76] G. Franchetti, S. Gilardoni, A. Huschauer, F. Schmidt, and R. Wasef, Space charge effects on the third order coupled resonance, Phys. Rev. Accel. Beams 20, 081006 (2017).

[77] A. Burov, Convective instabilities of bunched beams with space charge, Phys. Rev. Accel. Beams 22, 034202 (2019).

[78] G. Franchetti, Space charge, nonlinear resonances, and beam loss, J. Instrum. 15, P07018 (2020).

[79] J. Qiang, Advances in the simulation of space-charge effects, J. Instrum. 15, P07028 (2020).

[80] I. Hofmann, Revisiting coherent and incoherent resonances with space charge, J. Instrum. 15, P07016 (2020).

[81] V. Kornilov, A. Oeftiger, O. Boine-Frankenheim, V. Chetvertkova, S. Sorge, and P. Spiller, Beam quality and beam loss predictions with space charge for SIS100, J. Instrum. 15, P07020 (2020).

[82] I. Hofmann, A. Oeftiger, and O. Boine-Frankenheim, Selfconsistent long-term dynamics of space charge driven resonances in 2D and 3D, Phys. Rev. Accel. Beams 24, 024201 (2021).

[83] C. Moore, C. Curtis, J. Lackey, C. Owen, C. Ankenbrandt, R. Gerig, and S. Pruss, Dependence of the emittances of the Fermilab injectors on intensity, IEEE Trans. Nucl. Sci. 28, 3000 (1981).

[84] W. Chou, A. Drozhdin, P. Lucas, and F. Ostiguy, Fermilab Booster modeling and space charge study, in Proceedings of the 2003 Particle Accelerator Conference (IEEE, New York, 2003), Vol. 5, pp. 2925-2927.

[85] C. Ankenbrandt and S. Holmes, Limits on the transverse phase space density in the Fermilab Booster, Technical Report, Fermi National Accelerator Laboratory, 1987.

[86] S. D. Holmes, Design criteria and performance goals for the Fermilab Main Injector, Part. Accel. 58, 39 (1997).

[87] W. S. Graves, V. Bharadwaj, and D. McGinnis, A nondestructive fast beam profile monitor, Nucl. Instrum. Methods Phys. Res., Sect. A 364, 13 (1995).

[88] J. Amundson, J. Lackey, P. Spentzouris, G. Jungman, and L. Spentzouris, Calibration of the Fermilab Booster ionization profile monitor, Phys. Rev. ST Accel. Beams 6, 102801 (2003).

[89] C. Bhat, $\gamma_{t}$-jump in Booster during PIP-II era, Report No. Beams-doc-8735-v1, 2020, Fermilab internal note.

[90] P. Derwent, Implementation of BLonD for Booster simulations, Report No. Beams-doc-8690-v1, 2020, Fermilab internal note. 
[91] V. Kapin et al., Study of two-stage collimation system in Fermilab Booster, Report No. Beams-doc-5519-v1, 2017, Fermilab internal note.

[92] V. D. Shiltsev, Electron Lenses for Super-Colliders (Springer, New York, 2015).

[93] V. Danilov and S. Nagaitsev, Nonlinear accelerator lattices with one and two analytic invariants, Phys. Rev. ST Accel. Beams 13, 084002 (2010).
[94] S. Antipov, D. Broemmelsiek, D. Bruhwiler, D. Edstrom, E. Harms, V. Lebedev, J. Leibfritz, S. Nagaitsev, C.-S. Park, H. Piekarz et al., IOTA (Integrable Optics Test Accelerator): Facility and experimental beam physics program, J. Instrum. 12, T03002 (2017). 